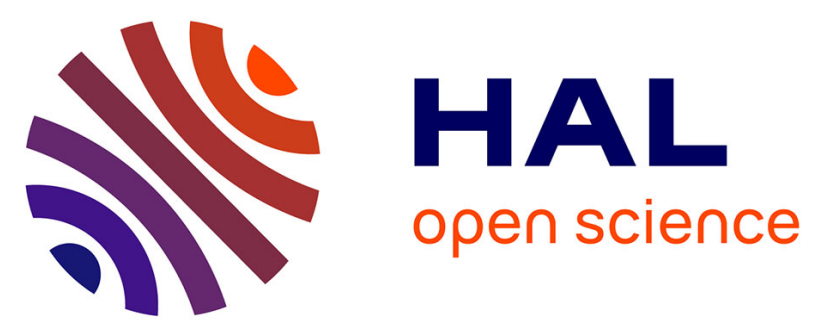

\title{
Therapeutic Benefits and Adverse Effects of Combined Pro-Angiogenic Gene Therapy in Mouse Critical Leg Ischemia.
}

Benoît Lebas, Julien Galley, Edith Renaud-Gabardos, Françoise Pujol, Françoise Lenfant, Barbara Garmy-Susini, Xavier Chaufour, Anne-Catherine Prats

\section{To cite this version:}

Benoît Lebas, Julien Galley, Edith Renaud-Gabardos, Françoise Pujol, Françoise Lenfant, et al.. Therapeutic Benefits and Adverse Effects of Combined Pro-Angiogenic Gene Therapy in Mouse Critical Leg Ischemia.: Combined Pro-Angiogenic Gene Therapy in Leg Ischemia. Annals of Vascular Surgery, 2016, pii: S0890-5096(16)31264-X. Epub ahead of print. 10.1016/j.avsg.2016.08.027 . inserm-01424778

\section{HAL Id: inserm-01424778 https://www.hal.inserm.fr/inserm-01424778}

Submitted on 2 Jan 2017

HAL is a multi-disciplinary open access archive for the deposit and dissemination of scientific research documents, whether they are published or not. The documents may come from teaching and research institutions in France or abroad, or from public or private research centers.
L'archive ouverte pluridisciplinaire HAL, est destinée au dépôt et à la diffusion de documents scientifiques de niveau recherche, publiés ou non, émanant des établissements d'enseignement et de recherche français ou étrangers, des laboratoires publics ou privés. 
Therapeutic Benefits and Adverse Effects of Combined Pro-Angiogenic Gene Therapy in Mouse Critical Leg Ischemia

Benoît Lebas ${ }^{1,2, *}$, Julien Galley ${ }^{1,2, *}$, Edith Renaud-Gabardos ${ }^{1, *}$, Françoise Pujol ${ }^{1}$, Françoise Lenfant $^{1}$, Barbara Garmy-Susini ${ }^{1}$, Xavier Chaufour ${ }^{1,2}$ and Anne-Catherine Prats ${ }^{1, \S}$

${ }^{1}$ I2MC, Université de Toulouse, Inserm, UPS, Toulouse, France.

${ }^{2}$ Centre Hospitalier Universitaire de Toulouse, Toulouse, France.

*Equal contribution

${ }^{\S}$ Corresponding author.

Contact Information: Anne-Catherine Prats, Institut des Maladies Métaboliques et Cardiovasculaires, 1, Avenue Jean Poulhes, BP 84225, 31432 Toulouse cedex 4, France (email: Anne-Catherine.Prats@inserm.fr)

Running title: Combined Pro-Angiogenic Gene Therapy in Leg Ischemia

Keywords: critical leg ischemia/ therapeutic angiogenesis/ combined gene therapy/ AAV/ FGF2/ Cyr61 


\section{ABSTRACT}

Objective: Critical leg ischemia (CLI) represents the ultimate stage of peripheral arterial disease. Despite current surgery advances, patients with CLI have limited therapeutic options. Therapeutic angiogenesis thus appears as a powerful approach, aiming to stimulate vessel formation by angiogenic molecules administration. In this context, combined gene therapy has proved the most efficient. The present study aims to compare, in a preclinical mouse model, the therapeutic benefit of a combination of two angiogenic factors FGF2 (fibroblast growth factor 2) and Cyr61 using plasmid and viral vectors, able to generate short or longterm transgene expression in the leg, respectively.

Methods: Two therapeutic genes, FGF2 and Cyr61, were introduced into IRES-based expression vectors (FGFiCyr) allowing co-expression of the two transgenes. The proangiogenic plasmid pC-FGFiCyr was assessed by intramuscular administration followed by electrotransfer into ischemic legs. To generate long-term transgene expression, the FGFiCyr bicistronic cassette was introduced into an adeno-associated virus-derived vector (rAAV). The rAAV treatment was performed either before, or immediately after, surgery. Therapeutic effects were analyzed by laser doppler imaging, clinical score and angiography.

Results: The plasmid pC-FGFiCyr improved revascularization, reperfusion and clinical score. Surprisingly, when AAV-FGFiCyr was injected 21 or 28 days before surgery, the proangiogenic rAAV was drastically deleterious on all measured parameters. In contrast, when administrated shortly after surgery, AAV-FGFiCyr generated therapeutic benefits, with a significantly better clinical score than after treatment with the plasmid.

Conclusions: Therapeutic effects of the angiogenic combination FGF2-Cyr61 is observed with short term transgene expression, but the treatment is significantly more efficient when a long-term expression viral vector is used. However, the rAAV FGFiCyr generated therapeutic benefit only when injected in an ischemic leg, whereas the same dose of rAAV exhibited 
deleterious effects when administrated to healthy animals. These data may contribute to the understanding of the moderate success of pro-angiogenic treatments in CLI gene therapy clinical assays.

\section{ABBREVIATIONS}

AAV: adeno-associated virus

CLI: critical leg ischemia

CMV: cytomegalovirus

FGF: fibroblast growth factor

HGF: hepatocyte growth factor

IRES: internal ribosome entry site

LDI: laser Doppler imaging

PAD: peripheral arterial disease

VEGF: vascular endothelial growth factor

\section{1/ INTRODUCTION}

Critical leg ischemia (CLI) represents the ultimate stage of peripheral arterial disease (PAD), with an incidence between 500 and 1000/million of inhabitants in European and NorthAmerican population. This pathology touches men three times more often than women. Despite the progress of surgical and endovascular techniques, patients with CLI have limited therapeutic options, one of which is often amputation.

In such a context, therapeutic angiogenesis appears to be a powerful approach, aiming to stimulate the formation of mature vessels by the administration of angiogenic molecules. Several studies report the use of gene therapy to generate angiogenesis (1). In that context, angiogenic factors including vascular endothelial growth factor (VEGFA), fibroblast growth 
factors 1 and 2 (FGF1 and FGF2) or hepatocyte growth factor (HGF) have been tested in clinical trials. The therapeutic benefits have been variable, HGF being the most successful but still not sufficient to restablish the vascular network in ischemic limbs (2).

Currently, clinical assays of gene therapy of CLI use plasmids or adenoviral vectors delivered by intramuscular or intra-arterial administration. Plasmids exhibit the disadvantage of transient expression, resulting in a low gene transfer efficiency, whereas adenoviral vectors are highly immunogenic. Such defaults suggest that the use of adeno-associated virus (AAV) derived vector could result in better benefits. Recombinant AAVs (rAAV) have been used successfully in preclinical assays in a rabbit leg ischemia model (1).

An attractive approach to improve the benefits of therapeutic angiogenesis in CLI gene therapy is provided by combined gene therapy. In a physiological situation, the development of functional vessels requires cooperation between several angiogenic growth factors, whereas the currently reported gene therapy approaches use only one angiogenic growth factor. This may generate an imbalance in the ratio of the different angiogenic growth factors necessary to stimulate formation of functional blood vessels. The concept of combined gene therapy has been validated by several studies (3). A few years ago, we showed a synergistic effect of FGF2 and Cyr61 in the treatment of mouse leg ischemia, using an IRES-based bicistronic vector (FGFiCyr). This previous report has shown that plasmid electrotransfer allows a more efficient therapeutic angiogenesis as well as a decrease in clinical signs when FGF2 and Cyr61 are co-expressed at low doses, compared to moderate effect of these growth factors alone, although expressed at high doses (4). In addition to these therapeutic benefits, the bicistronic plasmid generates no side effects on tumoral angiogenesis, in contrast to the monocistronic vector expressing huge doses of Cyr61.

The genetic background has a major influence on the mouse ability to respond to ischemia and generate physiological angiogenesis and arteriogenesis. Femoral artery ligation has only 
few consequences on wild type C57BL/6 mouse lineage. Limb complete reperfusion is restored between three and seven days after surgery (5). In an attempt to mimic human atherosclerosis, hypercholesterolemic $\mathrm{C} 57 \mathrm{BL} / 6$ mice have been generated by homozygous knock down of apoliprotein E (ApoE) (5). That model was used to show the synergistic effect of FGF2 and Cyr61 in the study mentioned above (4). However, the BALB/c model has been characterized for its sensitivity to ischemia associated to incomplete ability of ischemic leg reperfusion, thus this model was chosen in the present study to assess the efficiency of the FGFiCyr cassette on therapeutic angiogenesis (6).

In an attempt to compare short and long-term transgene expression, the FGFiCyr bicistronic cassette was introduced into an AAV-derived vector (rAAV). The therapeutic potential of the viral vector was compared to that of the plasmid. Different times of injection before or just after surgery were analyzed (Fig. 1).

\section{2/ MATERIAL AND METHODS}

\section{1/ Plasmids}

Bicistronic plasmids pC-FGFiCyr and pC-RiL have been described previously (4). Both contain the CMV promoter and the FGF1 IRES. pC-FGFiCyr codes for FGF2 and Cyr61, whereas pC-RiL codes for renilla and firefly luciferases. The AAV vectors were constructed by transferring the bicistronic cassettes FGFiCyr and RiL into the pAAV-MCS plasmid as previously described (7).

Naked plasmid DNA (50 $\mu \mathrm{g}$ in $30 \mu \mathrm{l}$ of sodium chloride solution $0.9 \%$ ) was injected (300 U, Myjector U-100 insulin, Terumo) into the tibialis anterior muscle and electrotransferred as previously described using an ECM 830 electroporator (BTX Division of Genetronics, Inc., San Diego, CA USA) . 
AAV production was performed by the vectorology plateau of Inserm UMR1037. Serotype $1 / 2$ rAAVs were produced using the tri-transfection procedure using the plasmids pHelper (Stratagene) and pDP1rs (Plasmidfactory), and injected in the mice tibialis anterior and gastrocnemius skeletal muscles (7). Vector titers were $3.9 \times 10^{11} \mathrm{pfu} / \mathrm{mL}$ for AAV-RiL and 0.6 to $1.2 \times 10^{13} \mathrm{pfu} / \mathrm{mL}$ for AAV-FGFiCyr. rAAV administration to each mouse $(100 \mu \mathrm{L})$ was divided into four injections: $20 \mu \mathrm{L}$ were injected in each tibialis anterior and $30 \mu \mathrm{L}$ in each gastrocnemius.

\section{3/ Animal experimentation}

Animal experiments were conducted in accordance with the recommendations of the 'European Convention for the protection of vertebrate animals used for experimental and other scientific purposes'. All animal experiments were performed according to the INSERM IACUC guidelines for laboratory animals husbandry and have been approved by the "Comité d'Ethique de la Chambre Professionnelle du Conseil Midi-Pyrénées" (protocol No MP/13/27/03/12). C57BL/6 and BALB/c JRJ 10-14 weeks old male mice were used (Janvier, Le Genest St Isle, France).

\section{4/ Ischemic leg model}

We used the murine leg ischemia model described previously . Surgery was performed under general anaesthesia was achieved by intraperitoneal injection of Ketamine $(125 \mathrm{mg} / \mathrm{kg}$ body weight) and Xylazine (10 mg/kg body weight) solution. The femoral artery was released on its whole length and ligated with Prolène ${ }^{\circledR}(8 / 0$ or $7 / 0)$. We performed ligation-exeresis of the whole femoral artery (Protocol 1, 2 and 4), or double arterial ligation of the common femoral artery and the sapheno-popliteal junction, the artery being cut between the two ligations (Protocol 3). Skin incision was closed using Prolène 6/0 ${ }^{\circledR}$. Animals were clinically followed every day. 


\section{5/ Laser Doppler perfusion imaging}

Blood flow in both ischemic and nonischemic hindlimbs was measured as previously until day 21 using a PeriScan System blood perfusion monitor laser Doppler equipment (Laser Doppler perfusion imaging system; Perimed, Stockholm, Sweden) (4). Results are expressed as the ratio of perfusion in the ischemic limb versus nonischemic hindlimb.

\section{6/ Angiography}

Angiographic analysis of collateral vessel development in the ischemic limb was performed by angiography on anesthetized animals as previously described (4). Briefly, after abdominal aorta exposure, a 24G polyethylene catheter (BD, Franklin Lakes, NJ) was used to cannulate the aorta, $5 \mathrm{ml}$ phosphate-buffered saline was injected, followed by $0.5-0.8 \mathrm{ml}$ contrast medium (Micropaque, barium sulfate $1 \mathrm{~g} / \mathrm{ml}$; Guerbet, Roissy, France). Angiograms were generated with a Faxitron X-Ray imager (MX-20; Faxitron X-Ray, Lincolnshire, IL). Pixel size was $48 \mu \mathrm{m}$. Films were exposed 10 seconds at $22 \mathrm{kV}$, then numerized for quantitative evaluation using image analysis software Morpho Expert (Explora Nova, La Rochelle, France).

\section{7/ Statistical analysis}

All statistical analyses were performed using a two-tailed Student's t-test or one-way ANOVA and are expressed as mean + - standard deviation, ${ }^{*} \mathrm{p}<0.05,{ }^{*} \mathrm{p}<0.01, * * *<0.001$.

\section{3/ RESULTS}

3.1/ Therapeutic benefit of BALB/c treatment with the bicistronic plasmid $p C-F G F i C y r$. 
Before using viral vectors in an attempt to obtain a long-term treatment, the bicistronic plasmid pC-FGFiCyr, as well as a control plasmid, was administrated to mice just before ligation-exeresis surgery, in order to analyse on BALB/c mice the therapeutic effect of this vector, previously used on C57BL/6 ApoE-/- (4) (Fig. 1A, protocol 1). Plasmid pC-RiL was used as a control. A clinical score of 0 to 6 was established according to the criteria described in Table 1. Following pC-FGFiCyr injection, the clinical score was improved at day 21 from 3.3 to 2.6 (Fig. 2A). LDI (Laser Doppler Imaging) analysis revealed a more important and faster reperfusion of the ischemic leg in the treated group $(n=6)$ compared to the control group $(n=7)$, from day 7 to day 21 after surgery (Fig. 2B and 2C). Moreover, angiography data showed an increase of all parameters: vessel area, thickness, length and number of segments (Fig. 2D and 2E).

These data validated the therapeutic effect of the FGFiCyr transgene in the BALB/c genetic background.

\section{2/ Adverse effect of bicistronic AAV-FGFiCyr administrated prior to surgery.}

The rAAVs are currently the most efficient vectors for gene transfer into muscle, and allow long-term expression of transgenes although not integrated in the host genome. The FGFiCyr cassette was transferred into an AAV vector (AAV-FGFiCyr).

The rAAVs are known to reach maximal transgene expression only two or three weeks after injection (8). Thus, in order to ensure a maximal vector expression at the beginning of ischemia, AAV-FGFiCyr and control rAAV were injected into tibialis anterior muscles, 21 or 28 days before surgery (Fig. 1B and 1C, protocols 2 and 3). Unexpectedly, the protocol 2 had to be stopped at day 10 after surgery, due to extreme clinical pain (evaluated according to behavior and physiological criteria) of the AAV-FGFiCyr group with a death at day 7 in this group. The LDI analysis showed no benefit for the treated group, whereas the clinical score was worse for the treated group (3.9) than for the control (3.1) (Fig. 3A and 3B). No benefit 
of the pro-angiogenic treatment was observed on the angiography parameters, despite a strong transgene expression detected by Western blot (Fig. 3C and 3D, Fig. S1).

When vector injection was performed 28 days before surgery (protocol 3), despite the less severe surgery the experiment had again to be stopped at day 10 after surgery, due to clinical pain. Here, the clinical score was significantly worse in the treated group (4.6) than in the control (2.2), while reperfusion did not vary (Fig. 4A and 4B). In addition angiography parameters showed a strong decrease of vessel area, length and segments (Fig. 4C and 4D).

Thus, these data showed an unexpected deleterious effect of the pro-angiogenic treatment with AAV-FGFiCyr, when injected several weeks prior to surgery. This adverse effect was stronger when AAV was injected 28 days (protocol 3), versus 21 days before surgery (protocol 2).

\section{3/ Therapeutic benefit of AAV-FGFiCyr administrated after surgery.}

The deleterious effect of AAV-FGFiCyr increased when the vector was injected earlier before surgery (28 versus 21 days, Fig. 3 and 4), suggesting that the time of injection may have an impact on the therapeutic effect of the vector. Thus, a new protocol was designed where the same doses of rAAVs were injected immediately after surgery (Fig. 1D, protocol 4). In this protocol, surgery had to be done in the A2 safety animal facility, preventing the use of the LDI apparatus (located outside). Here, no clinical pain was observed and the groups could be followed until day 21 , as for treatment with plasmid. Interestingly, the clinical score was significantly improved (1.8) (Fig. 5A). In addition, angiography revealed a significant increase of vessel area (Fig. 5B and 5C).

These data showed a significant therapeutic benefit of AAV-FGFiCyr, when injected immediately after surgery, with a significant clinical score improvement, superior to that observed with the plasmid pC-FGFiCyr. 


\section{4/ DISCUSSION}

These data show that combined gene therapy in a mouse model of CLI using an rAAV expressing FGF2 and Cyr61, can generate opposite effects, depending on the timing of the injection, either before or after surgery. The pro-angiogenic rAAV exhibited an important therapeutic benefit when injected just after surgery, while it was drastically deleterious when administrated on healthy animals several weeks before surgery.

The cooperative effect of FGF2 and Cyr61, previously reported in C57BL/6 ApoE -/- was also observed in BALB/c mice with the same bicistronic IRES-based plasmid (4). This shows that the efficiency of the vector pC-FGFiCyr is not dependent on the genetic background, suggesting that its therapeutic effect should also be generalizable to other mammalian species. Therefore, this pro-angiogenic vector may be an interesting candidate for a clinical trial in the future.

This mouse model of leg ischemia encounters an important limitation, as it does not really mimic critical limb ischemia in humans: it is a model of acute ischemia whereas the human pathology is chronic ischemia. This issue could be addressed using a mouse model recently validated by Lejay et al, using sequential femoral and iliac ligatures in Swiss mice at day 0 and day 4 , respectively (9).

The pivotal issue raised by our data is the strong difference of the AAV-FGFiCyr therapeutic effect depending on the date of administration relative to surgery. The therapeutic vector AAV-FGFiCyr is clearly beneficial on both clinical score and therapeutic angiogenesis when injected immediately post-surgery, whereas it is drastically deleterious when injected several weeks before surgery. This adverse effect is still stronger when injection occurs 28 days versus 21 days before surgery. We propose several hypotheses to explain such an unexpected effect.

A first hypothesis could be that rAAV injection might generate inflammation in the injected leg, resulting in an exacerbated sensitivity to the surgery. However, in such a case, the control 
rAAV should provide the same clinical profile as the therapeutic AAV. Our data suggest that the deleterious effect of AAV-FGFiCyr is related to the angiogenic transgenes FGF2 and Cyr61. This could be due to high doses of growth factors produced by rAAV. However, in a previous report where we used vectors expressing huge amounts of FGF2 or Cyr61, we never observed any deleterious effect of the treatment, although the therapeutic benefit was less important than with the growth factor combination (4). Our data suggest that FGF2 and Cyr61 may have different effects, depending on the basal state of the individual. In particular, the effect of Cyr61 is dependent on its interaction with integrins. When interacting with $\alpha_{\mathrm{v}} \beta_{3}$ present on endothelial cells, it induces angiogenesis whereas its interaction with $\alpha_{6} \beta_{1}$ expressed by fibroblasts results in apoptosis and cell senescence (10). Integrins may vary in ischemic versus normal legs.

Another interesting hypothesis is that overexpression of angiogenic factors before surgery would disturb the angiogenic balance (11). Treatment of healthy animals with pro-angiogenic factors may trigger expression of anti-angiogenic factors, whose presence, at the time of surgery, would prevent the normal angiogenic response to ischemia. Further investigation is needed to check such a possibility. Nevertheless, these observations indicate that the effect of pro-angiogenic treatments may be dependent on the basal state of the individual. This might also provide an avenue to explain the lack of efficiency of pro-angiogenic treatments in clinical assays of critical limb ischemia gene therapy, contrasting with beneficial effects of the treatments observed on animal models.

\section{5/ CONCLUSION}

In conclusion, our data show that an rAAV co-expressing FGF2 and Cyr61 exhibits a significant therapeutic benefit, superior to that of the plasmid vector expressing the same transgenes. However, this benefit is observed only when the pro-angiogenic rAAV is injected in an ischemic situation. Strikingly, the same dose of rAAV was severely deleterious when 
administered to healthy animals several weeks before surgery. These observations indicate that the effect of pro-angiogenic treatments may be dependent on the basal state of the individual.

\section{6/ ACKNOWLEDGMENTS}

Our thanks go to Y. Barreira, D. Calise and K. Pestourie from the Inserm US006 platform ANEXPLO Genotoul (Toulouse, France) and Loïc van den Berghe from the Inserm UMR1037 vectorology plateau (Toulouse). We also thank F. Cabon and H. Prats for their helpful discussion and M. Tortolano for English proofreading.

This work was supported by Fondation de l'Avenir, Association Française contre les Myopathies (AFM), Association pour la Recherche sur le Cancer, Région Midi-Pyrénées, European funding (REFBIO). E.R.G. had a fellowship from AFM.

\section{7/ REFERENCES}

1. Mughal NA, Russell DA, Ponnambalam S, Homer-Vanniasinkam S. Gene therapy in the treatment of peripheral arterial disease. The British journal of surgery 2012;99:6-15.

2. Madonna R, Rokosh G. Insights into gene therapy for critical limb ischemia: the devil is in the details. Vascular pharmacology 2012;57:10-4.

3. Renaud-Gabardos E., Hantelys F., Morfoisse F., Chaufour X., Garmy-Susini B., Prats A.-C. Internal ribosome entry site-based based vectors for combined gene therapy. World journal of experimental medicine, 2015;5:11-20.

4. Rayssac A, Neveu C, Pucelle M, Van den Berghe L, Prado-Lourenco L, Arnal JF, et al. IRES-based vector coexpressing FGF2 and Cyr61 provides synergistic and safe therapeutics of lower limb ischemia. Molecular therapy : the journal of the American Society of Gene Therapy 2009;17:2010-9. 
5. Couffinhal T, Silver M, Kearney M, Sullivan A, Witzenbichler B, Magner M, et al. Impaired collateral vessel development associated with reduced expression of vascular endothelial growth factor in ApoE-/- mice. Circulation 1999;99:3188-98.

6. Helisch A, Wagner S, Khan N, Drinane M, Wolfram S, Heil M, et al. Impact of mouse strain differences in innate hindlimb collateral vasculature. Arteriosclerosis, thrombosis, and vascular biology 2006;26:520-6.

7. Prats AC, Van den Berghe L, Rayssac A, Ainaoui N, Morfoisse F, Pujol F, et al. CXCL4L1-fibstatin cooperation inhibits tumor angiogenesis, lymphangiogenesis and metastasis. Microvascular research 2013;89:25-33.

8. Delluc-Clavieres A, Le Bec C, Van den Berghe L, Conte C, Allo V, Danos O, et al. Efficient gene transfer in skeletal muscle with AAV-derived bicistronic vector using the FGF1 IRES. Gene therapy 2008;15:1090-8.

9. Lejay A, Choquet P, Thaveau F, Singh F, Schlagowski A, Charles AL, et al. A new murine model of sustainable and durable chronic critical limb ischemia fairly mimicking human pathology. European journal of vascular and endovascular surgery : the official journal of the European Society for Vascular Surgery 2015;49:205-12.

10. Lau LF. CCN1/CYR61: the very model of a modern matricellular protein. Cellular and molecular life sciences : CMLS 2011;68:3149-63.

11. Folkman J. Angiogenesis in cancer, vascular, rheumatoid and other disease. Nature medicine 1995; 1:27-31. 


\section{8/ FIGURE LEGENDS}

Figure 1. Schema of the four protocols used in the study.

The protocols used in the following figures are schematized (A to D). The black horizontal arrows show the time in days. Day 0 (D0) corresponds to surgery. Times of vector injection, clinical score and angiography are indicated with vertical grey arrows. The different times of Laser Doppler Imaging are indicated (LDI).

Figure 2. Treatment of ischemic BALB/c mice with the bicistronic plasmid pC-FGFiCyr. Plasmid pC-FGFiCyr and pC-RiL DNAs were electrotransferred as previously into tibialis anterior muscle just before ligation-exeresis surgery (Fig. 1A, protocol 1) (4).

A. Clinical score was analysed at day 21 after surgery.

B and C. Perfusion was followed by LDI until day 21 post surgery and quantified (B). Imaging is presented for a representative individual. The relative perfusion value is obtained from the ratio of ischemic leg/non ischemic leg for each individual.

D and E. Angiography was performed (D) and vessels quantified using Morphoexpert (E). Different parameters were quantified: vessel area, average thickness, total length and segment number (mean + - standard deviation, ${ }^{*} \mathrm{p}<0.05, * * *<0.001$ ).

Figure 3. Treatment of ischemic BALB/c mice with the bicistronic vector AAV-FGFiCyr administrated 21 days before surgery.

AAV-FGFiCyr and AAV-RiL were injected into tibialis anterior muscle. 21 days later, ligature-exeresis surgery was performed and mice were followed as above (Fig. 1B, protocol 2). The protocol had to be stopped at day 10, as the clinical pain of the AAV-FGFiCyr group 
was too strong. Clinical score (A), LDI (B) and angiography (C) analyses were performed as in Figure 2.

Figure 4. Treatment of ischemic BALB/c mice with the bicistronic vector AAV-FGFiCyr administrated 28 days before surgery.

AAV-FGFiCyr and AAV-RiL were injected into tibialis anterior muscle. 28 days later, double ligation surgery was performed and mice were followed as above (Fig. 1C, protocol 3). The protocol had to be stopped at day 10, as the clinical pain of the AAV-FGFiCyr group was too strong. Clinical score (A), LDI (B) and angiography (C and D) analyses were performed as in Figure 2 (mean +- standard deviation, $* * \mathrm{p}<0.01, * * *<0.001)$.

Figure 5. Treatment of ischemic BALB/c mice with the bicistronic vector AAV-FGFiCyr administrated after surgery.

Ligation-exeresis surgery was performed on BALB/c mice. Then, on the same day, AAVFGFiCyr and AAV-RiL were injected into tibialis anterior muscle (Fig. 1D, protocol 4). AAV-FGFiCyr was injected at two doses. Clinical score (A) and angiography (B and C) analyses were performed as in Figure 2 (mean + - standard deviation, $* p<0.05$, $* * *<0.001)$. 


\begin{tabular}{|ll|}
\hline Clinical Score & Lesions observed \\
\hline 0 & No clinical sign of ischemia \\
1 & Limb cyanosis \\
2 & Lesions localised to toes : ulcer, necrosis or toe amputation \\
3 & Lesions extending to forefoot : ulcer or necrosis \\
4 & Transmetatarsal amputation \\
5 & Transtibial amputation or necrosis extending to tibia \\
6 & Trophic lesions extending to crural segment \\
\hline
\end{tabular}


Lebas Figure 1

A

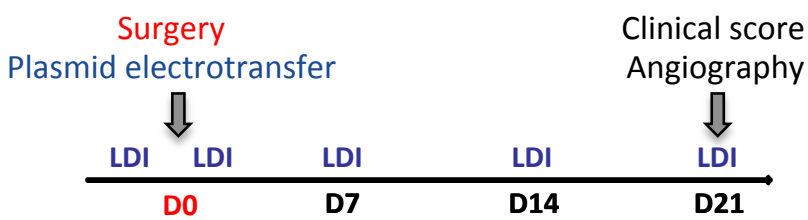

B

Protocol 2

\begin{tabular}{llllll}
$\stackrel{\Omega}{\text { D-21 }}$ & LDI & LDI & LDI & \\
\hline & D0 & D7 & D10
\end{tabular}

C

rAAV injection

Protocol 3

D

Protocol 4

Clinical score

Surgery Angiography

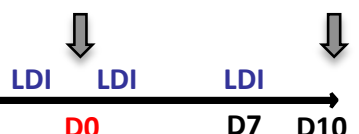

Surgery

rAAV injection

$\sqrt{ }$
Clinical score

Angiography

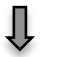

D0

D21 
Lebas Figure 2

A

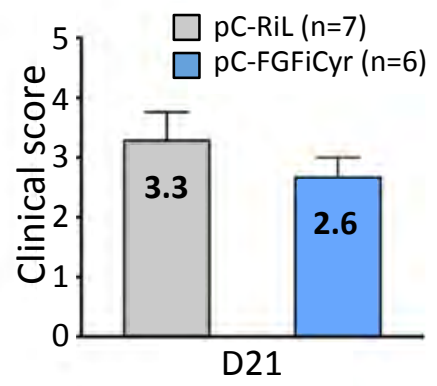

B

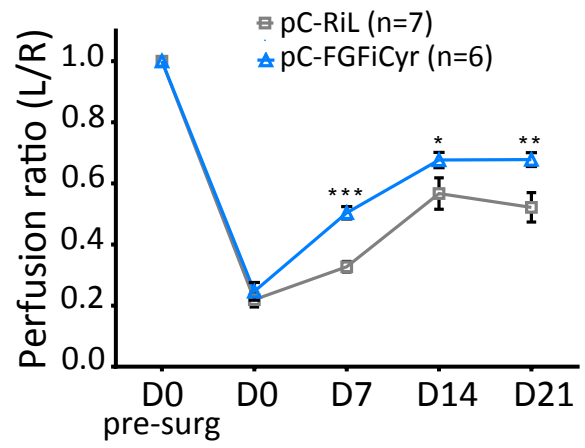

Time (days)

C

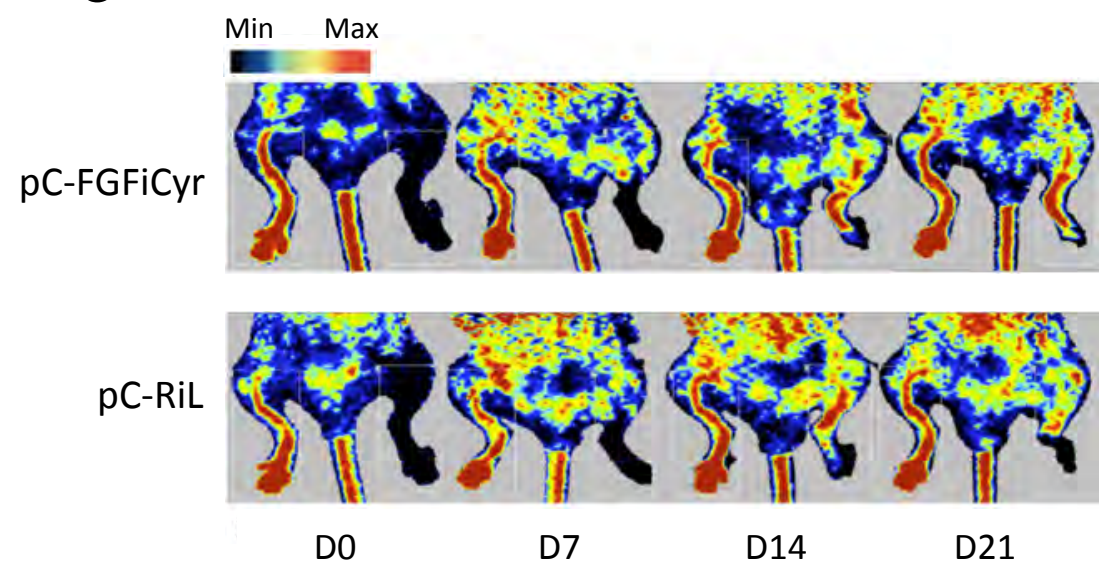

D
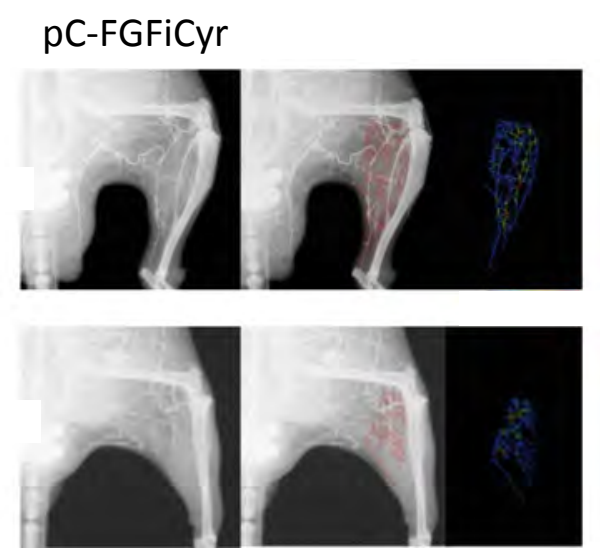

pC-RiL
E

$\square$ pC-RiL (n=6)

$\square$ pC-FGFiCyr (n=6)
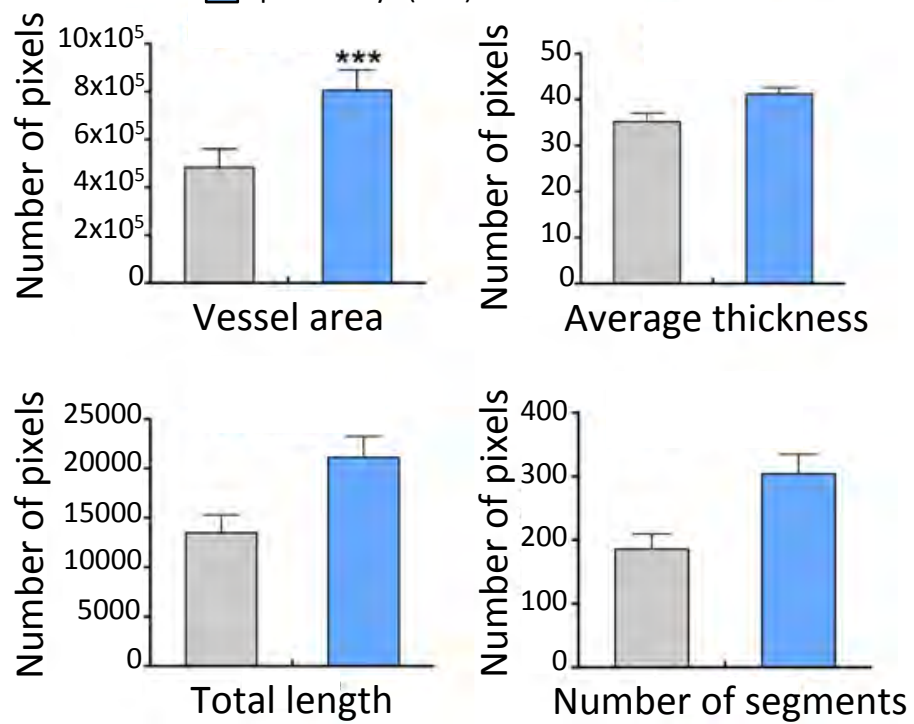
Lebas Figure 3

A

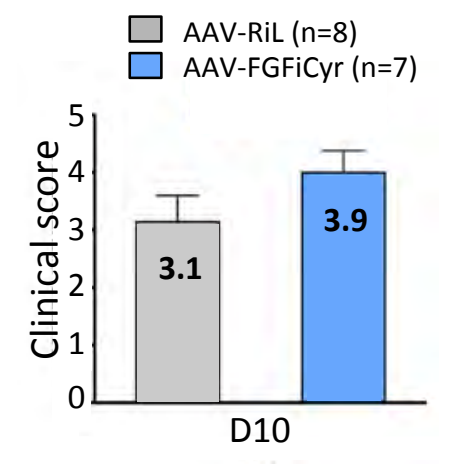

C
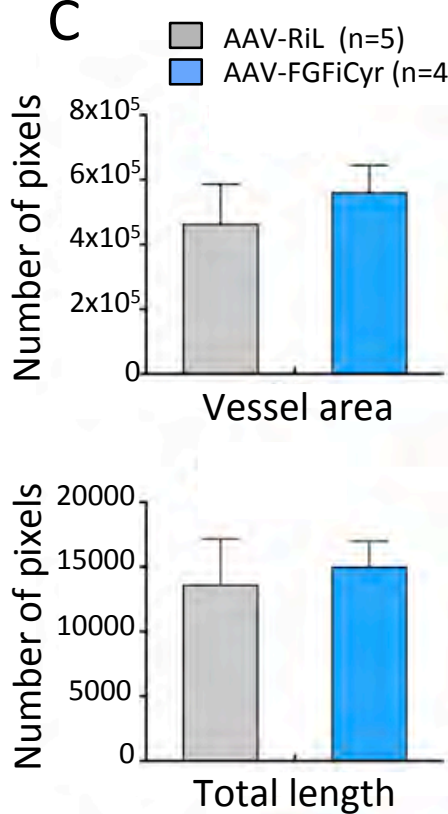

B

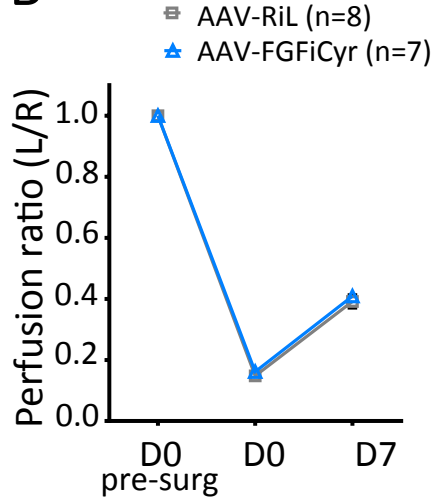

Time (days)
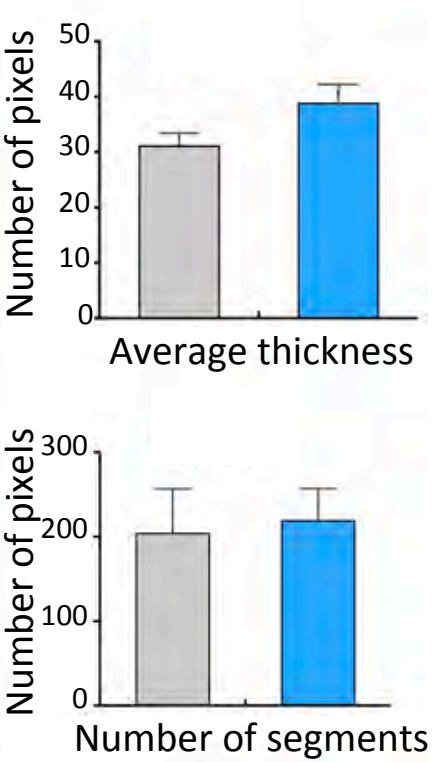
Lebas Figure 4
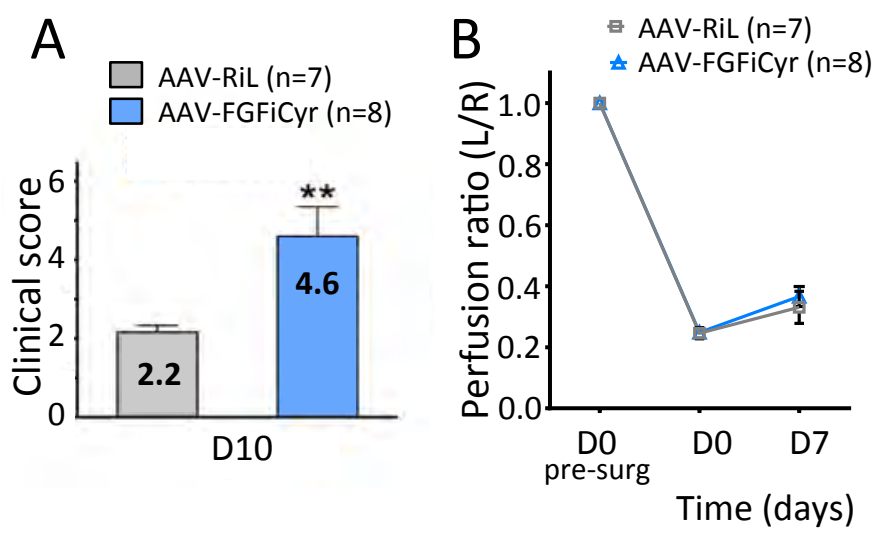

C
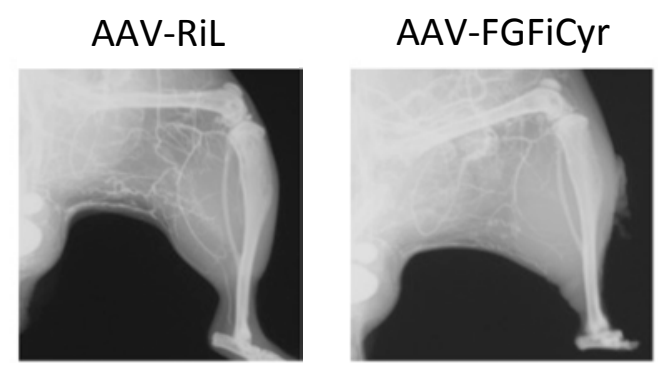

D
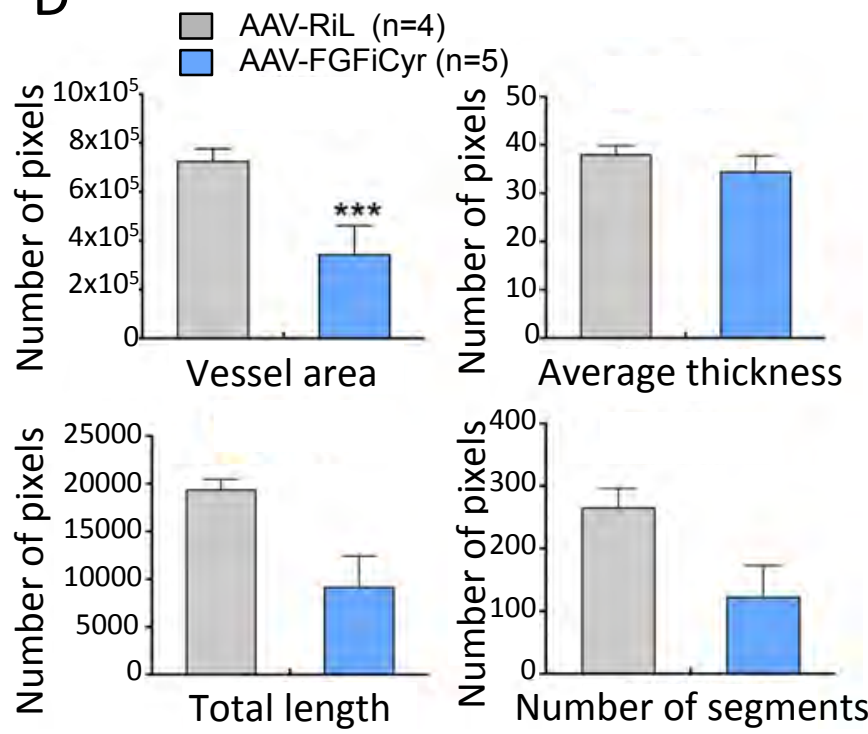
A

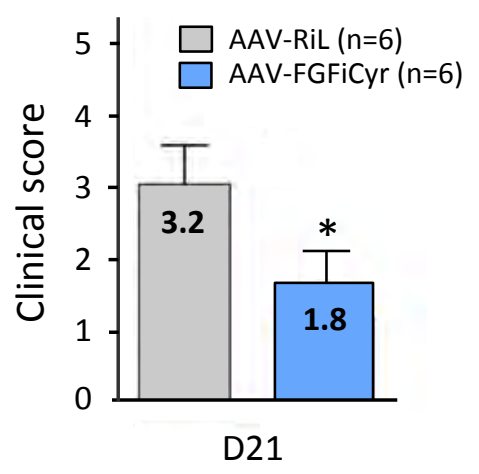

B

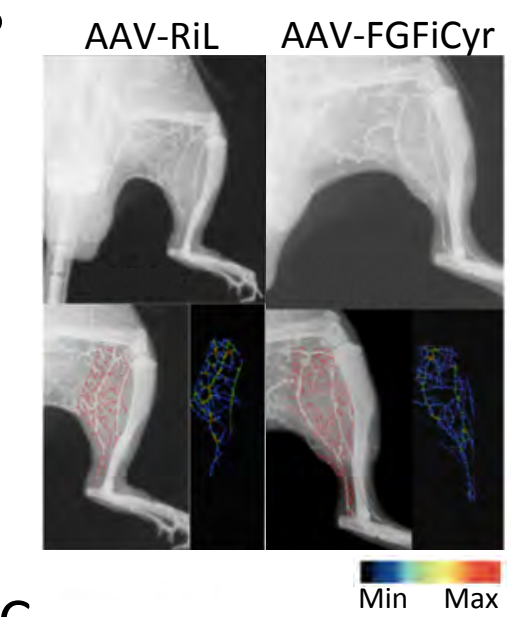

C

Min Max

AAV-RiL $(n=6)$

$\square$ AAV-FGFiCyr $(n=6)$

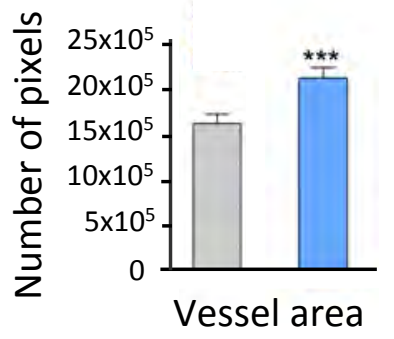

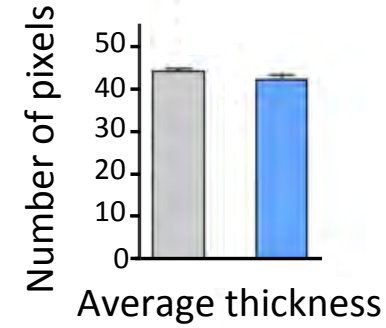
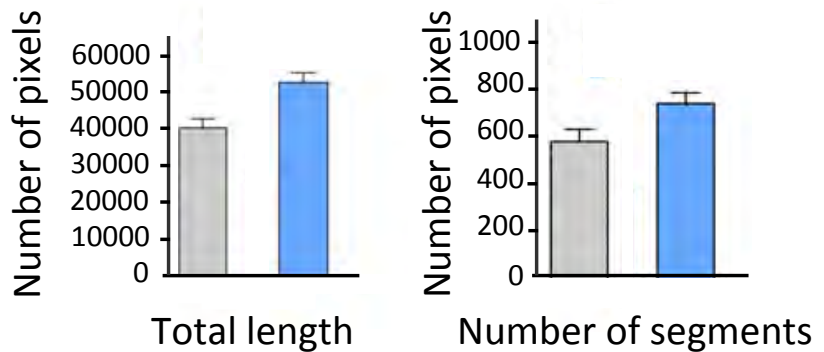
Lebas Figure S1

A

\section{Protocol 2}

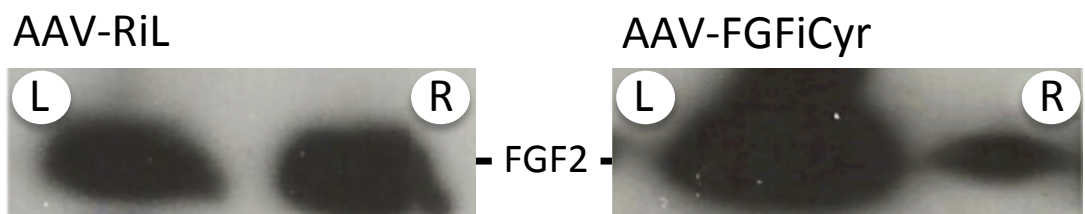

B

\section{Protocol 4}

AAV-RiL

AAV-FGFiCyr

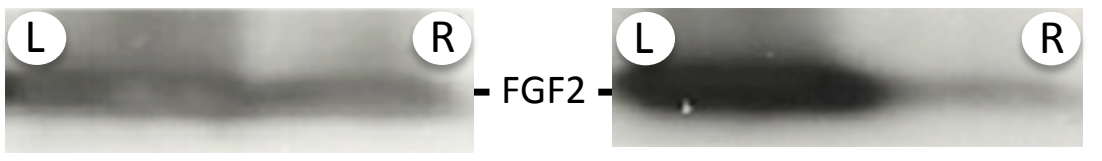

Figure S1. FGF2 expression following treatment of ischemic mice with the rAAVs. FGF2 expression was analyzed by Western immunoblotting using anti-FGF2 antibody on muscle extracts in protocol 2 (injection 21 days before surgery)(A) and protocol 4 (injection just after surgery)(B).

Whole-muscle protein extracts (obtained after homogenization in Tissue Extraction Reagent I (Invitrogen, Carlsbad, CA, USA) of ischemic muscles) were analyzed as previously described (1): $150 \mu \mathrm{g}$ of protein per sample was separated on a $4-20 \%$ polyacrylamide gel (Pierce Biotechnology, Inc., Rockford, IL) and electroblotted on nitrocellulose membranes. Western immunoblotting was performed using 1/400 rabbit polyclonal anti-FGF2 (Santa Cruz, FGF-2 (147): sc-79).

For AAV-RiL (control vector) and AAV-FGFiCyr (therapeutic vector), FGF2 expression was analyzed in the left leg (injected) and in the right leg (non injected as a control). The non injected right leg shows endogenous FGF2 expression. 50 ug of proteins was loaded in each lane. For each protocol, the four lanes come from the same blot thus the same exposure.

1. Rayssac A, Neveu C, Pucelle M, Van den Berghe L, Prado-Lourenco L, Arnal JF, et al. IRES-based vector coexpressing FGF2 and Cyr61 provides synergistic and safe therapeutics of lower limb ischemia. Mol Ther 2009 Dec;17:2010-9. PubMed PMID: 19738600 